\title{
Development of galacto-oligosaccharide fortified cookies for gut health management of normal 2-5 years old children
}

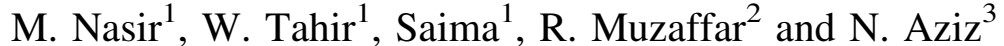 \\ ${ }^{1}$ Department of Food \& Nutrition, University of Veterinary \& Animal Sciences, Lahore, Pakistan, ${ }^{2}$ Department of Nutrition \\ \& Dietetics, Institute of Public Health, Lahore, Pakistan and ${ }^{3}$ Department of Social \& Pediatric, King Edward Medical \\ University, Lahore, Pakistan
}

Prebiotics (including galacto-oligosaccharides) are deliberated as non-digestible food ingredient that beneficially affects the host by selectively stimulating the growth and/or activity of one or a limited number of bacteria in the colon, and thus improve host health. They impart several important physiological effects depending on the composition and/or balance besides activities of beneficial microflora i.e. Bifidobacterium and Lactobacillus. ${ }^{(1)}$ Children who are fed on infant formula are more susceptible to infectious diseases than breast fed children. ${ }^{(2)}$

Keeping in view the need for the improvement of child health through improving the immunity, the present research project was designed. The study was conducted in three different phases. In the first phase, characterization of galacto-oligosaccharides (GOS) was performed. During the second phase blending of oligomate with all-purpose wheat flour at 12, 24, 36 and $48 \%$ (w/w) levels were executed to formulate prebiotic fortified-wheat-flour cookies at 1,2,3 and 4\%. The cookies thus prepared were physically and organoleptically evaluated for selection of optimum level of prebiotic fortification. During third stage, efficacy of GOS (Oligomate) fortified cookies were evaluated on forty five selected healthy children about 2-5 years old. Children were divided into three groups, A (control), B (treated with unfortified cookies per day) and C (treated with galacto-oligosaccharides fortified cookies per day) for 40 days. Urine and stool samples were analyzed at 0, 20 and 40 days of study. Weekly follow-up visits were scheduled and consisted of a detailed physical examination and other health and diet related information through pre-structured interviews and anthropometric measurements. The data obtained was statistically analyzed and significant results were observed for physical and chemical properties of oligomate.

\begin{tabular}{|c|c|c|c|c|c|c|c|}
\hline \multirow[b]{3}{*}{ Groups } & \multicolumn{6}{|c|}{ Study Interval } & \multirow[b]{3}{*}{ Mean } \\
\hline & \multicolumn{2}{|c|}{0 DAY } & \multicolumn{2}{|c|}{20 DAY } & \multicolumn{2}{|c|}{$40 \mathrm{DAY}$} & \\
\hline & Mean & $\mathrm{SD}$ & Mean & $\overline{\mathrm{SD}}$ & Mean & $\mathrm{SD}$ & \\
\hline Control & 7.60 & 1.18 & 7.53 & 1.45 & 7.61 & 1.14 & $7.58 \mathrm{~b}$ \\
\hline Unfortified cookies & 8.31 & 0.53 & 8.32 & 0.51 & 8.09 & 0.71 & $8.24 \mathrm{a}$ \\
\hline Fortified Cookies & 8.46 & 0.34 & 8.50 & 0.29 & 8.51 & 0.42 & $8.49 \mathrm{a}$ \\
\hline
\end{tabular}

Mean values of colony forming units for Lactobacillus acidophilus. (ANOVA, followed by Duncan Multiple Range test): $* P<0.05$.

Physical and chemical analysis of cookies also showed significant results however within the treatment non-significant results were observed as well as the storage study also showed non-significant results. The mean squares (2.53) for colony forming units and p-value $(0.014 *)$ for groups on various diets showed significant results. However, Mean squares for interval and the interaction between groups and study interval were non-significant. These results were in line with the study of Piirainen ${ }^{(3)}$ who evaluated the effects of galactooligosaccharide. Overall, prebiotic fortified cookies were found to be highly acceptable \& favorably impacted on gut microbioata

1. Tuohy KM, Rouzaud GC, Brück WM \& Gibson GR (2005) Curr Pharm Des 11, 75-90.

2. Fanaro S, Vigi V, Chierici R \& Boehm G (2003) Acta Paediatr 92, 634-5.

3. Piirainen L, Kekkonen RA, Kajander K et al. (2008) Ann Nutr Metab 52, 204-8. 